\title{
A PROBLEM OF P. A. SMITH
}

\section{HSIEN-CHUNG WANG}

In a paper in this Bulletin, ${ }^{1} \mathrm{P}$. A. Smith has mentioned the problem whether in a non-abelian Lie group $G$ there exists a non-countable proper subgroup everywhere dense in $G$. We can see that a negative answer to this problem is unlikely as the non-existence of such group implies the well known continuum hypothesis. It is the aim of the present short note to show that each separable, locally compact, nondiscrete metric group has a subgroup possessing the above properties.

Let $G$ be an abstract group and $S$ a subset of $G$. The least subgroup of $G$ which contains $S$ will be called the group closure of $S$ and denoted by $\operatorname{gcl}(S)$. Evidently, $\operatorname{gcl}(S)$ consists of all the finite products of the elements of $S$ and their inverses. It follows immediately that $S=\operatorname{gcl}(S)$ if and only if $S$ forms a subgroup of $G$.

Suppose $R$ to be a subset of $G$ and $p$ an element of $G$ such that $p$ does not belong to the group closure $\operatorname{gcl}(R)$ of $R$. Using Zorn's Theorem ${ }^{2}$ which is equivalent to the Axiom of Choice, we can construct a subset $H$ of $G$ having the following properties:

(i) $H$ forms a subgroup of $G$;

(ii) $H$ contains $R$ but does not contain $p$;

(iii) if $t$ is an element of $G$ not belonging to $H$, then $p$ is contained in the group closure $\operatorname{gcl}(H \cup t)$ of the union $H \cup t$. In general, there exists more than one such subgroup $H$. We shall call each of them a maximal subgroup including $R$ but excluding $p$.

Now let us consider a separable, locally compact, nondiscrete metric group $G$. We choose, in $G$, a countable everywhere dense subset $R$. The group closure $\operatorname{gcl}(R)$ of $R$ is also countable. However, the group $G$, being nondiscrete, is a perfect space. Therefore, $G$ must be non-countable, for otherwise it would be homeomorphic with the set of all rational numbers ${ }^{3}$ which is not locally compact. It follows that there exists an element $p$ of $G$ which does not belong to $\operatorname{gcl}(R)$. We can construct a maximal subgroup $H$ including $R$ but excluding $p$. Evidently, $H$ forms a proper, everywhere dense subgroup of $G$.

We shall show that $H$ is non-countable. For this purpose, let us

Received by the editors October 27, 1948.

1 P. A. Smith, Everywhere dense subgroups of a Lie group, Bull. Amer. Math. Soc. vol. 48 (1942) pp. 309-312.

2S. Leftschetz, Algebraic topology, Amer. Math. Soc. Colloquium Publications, vol. 27, New York, 1942, p. 5.

- W. Sierpinski, Introduction to general topology, Toronto, 1934, p. 107. 
consider equations of the form

$$
p=z^{m_{1}} h_{1} z^{m_{2}} h_{2} \cdots h_{s} z^{m_{0}+1}
$$

where $m_{i}(i=1,2, \cdots)$ denote integers, $h_{i}$ elements in $H$, and $z$ the unknown. The set $C$ of solutions of such an equation is closed in $G$, and from the maximal property of $H, C$ belongs to the complement $G-H$ of $H$. Since $H$ is everywhere dense, $C$ is nowhere dense.

Suppose $H$ to be countable. Then the aggregate of all equations of the form (1) is countable as well. Thus the union $Z=U C$ of all the possible $C$ 's is a set of the first category. Moreover, we can easily see that $Z$ coincides with the complement $G-H$ of $H$. On the other hand, $H$ is countable and $G$ perfect, so that $H$ is nowhere dense in $G$. It follows then that $G=H+Z$ is of the first category in itself. This contradicts the fact that $G$ is a locally compact metric space. Therefore, $H$ cannot be countable and we arrive at the following:

In any separable, locally compact, non-discrete metric group $G$, there always exists a non-countable proper subgroup filling $G$ densely. ${ }^{4}$

Institute of Mathematics, Academia Sinica

4 The author wishes to express his sincere thanks to Professor D. Montgomery for his valuable suggestions which enable the author to prove the theorem under much weaker condition for $G$ than a Lie group. 